\title{
Trigeminal neuralgia complicating a syndromic craniosynostosis treated with acupuncture: a case report
}

\begin{abstract}
Trigeminal neuralgia (TN) is the most common cranial neuralgia, defined as sudden, usually unilateral, severe, brief, stabbing, recurrent episodes of pain in the distribution of one or more branches of the trigeminal nerve; some patients experience also a degree of dull, burning, or tingling pain between attacks. TN may have a significant impact upon quality of life due to the associated depression and anxiety. TN's pain is generally managed with medical therapy alone, carbamazepine being the first-line medication. Pfeiffer syndrome is a rare inherited disorder associated with primary craniosynostosis, midface hypoplasia and other anatomical abnormalities. Acupuncture is a valid therapeutic option for painful conditions, including neuralgias. Hereby, we describe remission from trigeminal neuralgiarelated pain in a 37years old man with Pfeiffer Syndrome, after acupuncture.
\end{abstract}

Keywords: pfeiffer syndrome, traditional chinese medicine acupuncture, auriculotherapy, trigeminal neuralgia
Volume 13 Issue 5 - 2020

\author{
Carmelo Pasquale Guido,' Veronica \\ Santarlasci, ${ }^{2}$ Oreste Gallo, ${ }^{3}$ Rocco Domenico \\ Mediati, ${ }^{4}$ Glauco Cristofaro ${ }^{5}$ \\ 'Fior di Prugna Center for Complementary Medicine, Local \\ Health Unit, Italy \\ ${ }^{2}$ Department of Anesthesia,AOUC Careggi University Hospital, \\ Italy \\ ${ }^{3}$ Department of Experimental and Clinical Medicine, University \\ of Florence, Italy \\ ${ }^{4}$ Department of Palliative Care and Pain Therapy,AOUC Careggi \\ University Hospital, Italy \\ ${ }^{5}$ Department of Otorhinolaryngology Head and Neck Surgery, \\ AOUC Careggi University Hospital, Italy
}

\begin{abstract}
Correspondence: Carmelo P Guido, Fior di Prugna Center for Complementary Medicine, Local Health Unit, Central Tuscany, Via della Piazzuola, 68 - 50133 Florence, Italy, Tel+39 0556939240, Fax +39 0556939239,

Email carmelopasquale.guido@uslcentro.toscana.it
\end{abstract}

Received:September 24, 2020 | Published: October 08, 2020
Abbreviations: PS, pfeiffer syndrome; IASP, international association for the study of pain; TN, trigeminal neuralgia; UIM, unit of integrative medicine

\section{Introduction}

Trigeminal neuralgia (TN) is the most common cranial neuralgia, with an incidence of up to 5 per 100,000 and a prevalence of $0.07 \%$. The International Association for the Study of Pain (IASP) defines trigeminal neuralgia as "sudden, usually unilateral, severe, brief, stabbing, recurrent episodes of pain in the distribution of one or more branches of the trigeminal nerve". ${ }^{2}$ In patient's history two features define possible trigeminal neuralgia: paroxysmal pain, and a distribution consistent with that of the nerve. The trigeminal nerve has three branches: the ophthalmic, the maxillary, and the mandibular branch, being the latter the most commonly affected. In a majority of the patients, pain is evoked by non-noxious mechanical stimuli in specific "trigger zones." Some patients experience some degree of dull, burning, or tingling pain between attacks. ${ }^{3} \mathrm{TN}$ is classified in three etiologic categories: idiopathic, that occurs without apparent cause; classic, caused by nerve root vascular compression; secondary, consequence of a neurologic disease (e.g. a tumour or multiple sclerosis). Studies have reported a significant impact upon quality of life, the ability to perform daily tasks ${ }^{4}$ and social interactions, depression and anxiety. ${ }^{5} \mathrm{TN}$ is generally managed with medical therapy alone, carbamazepine being the first-line medication.

Pfeiffer syndrome (PS) is a rare autosomal dominant inherited disorder affecting about 1 in 100,000 births. It is associated with primary craniosynostosis, midface hypoplasia, broad thumbs and great toes, and soft tissue syndactyly of hands and feet of varying severity. ${ }^{6,7}$ Abnormal growth of the skull bones leads to the facial abnormalities defining the syndrome: proptosis, wide-set eyes, high forehead, underdeveloped maxilla, and beaked nose. Patients may manifest secondary nasal obstruction; usually due to midface hypoplasia. ${ }^{8}$ Staged surgeries are the recommended treatment for PS.

Acupuncture proved a valid therapeutic option for diverse conditions, including chronic diseases ${ }^{9}$ and neuropathic pain, ${ }^{10}$ as well as neuralgias. ${ }^{11}$ Hereby, we describe remission from trigeminal neuralgia-related pain in a 37years old man suffering from Pfeiffer Syndrome.

\section{Case presentation}

A 37years old Caucasian man with Pfeiffer Syndrome suffering from chronic, severe, reluctant trigeminal pain was referred to our Unit of Integrative Medicine (UIM). The patient was diagnosed with Pfeiffer Syndrome by the age of 2weeks by the human genetics department. The objective examination showed a craniosynostosis of metopic and coronary sutures with plagiocephaly, frontal flattening with hypoplasia of the orbital arches, turricephaly and syndactyly of hands and feet. During the first eighteen months cranium and plagio plastic were performed. By the age of five to twenty-two he underwent an orthodontic treatment. At the age of twenty-one and twenty-two he underwent Le Fort II and Le Fort III osteotomies, genioplasty, overbite and exorbitism correction, orbital ptosis correction and lipostructure after Coleman. During this period the patient started to report nasal obstruction, sleep apneas, snoring and frequent colds. At the age of 34 he began to complain of facial and nasal pain, treated with NSAIDs 
and ice during paroxysms. Three years later the patient turned to the Otolaryngology department. After the CT scan showed the presence of nasal polyps he underwent surgical removal: nevertheless, no improvement in pain was achieved.

At presentation in our UIM, the main symptoms consisted of unilateral (left) midfacial pain described as dull and oppressive, not pulsating, lasting hours to days and frequent relapses, with hyperalgesia of the left zygomatic region, sense of foreign body in the left nasal fossa and nasal obstruction. Pain become exacerbated in the last year with over-imposed intermittent episodes of burning or stabbing sensation lasting seconds to hours, reaching a VAS score of 10 in the acute phase. The neurological examination pointed out a reduction in superficial and deep sensitivity. Symptoms and signs were consistent with a neuropathic pain, by involvement of the second (maxillary) trigeminal branch. The planned treatment consisted of weekly sessions of Traditional Chinese Medicine acupuncture and auriculotherapy lasting 30minutes each. Disposable stainless steel needles (ø $0,25 \times 13 \mathrm{~mm} ; 0,25 \times 25 \mathrm{~mm}$, Hwato, China) were inserted into the skin by a more than 30 years experience acupuncturist and when appropriate manipulated for not longer than 1minute; the depth of needle insertion varied according to the site (face and ear 0.3-0.5 cun; hand and leg 0.5-2 cun). Unilateral (left) points chosen for the first session: EX-NH3 (Yintang), EX-NH8 (Bitong), EXNH5 (Taiyang), 1BL, 18SI, 8ST, 4LI. Before the first session and at the end of the treatment the patient filled the short form health survey SF-12 questionnaire, and every session before and after the acupuncture treatment the patient reported the VAS score. Having achieved almost complete relief from the symptoms by the end of the first session, reaching a VAS score of 1 starting from 5 (Figure 1A), following treatments focused on consolidation of these results. In the following sessions, points selection included left unilateral EX-NH3, EX-NH8, EX-NH5, 1UB, 18SI, 8ST, LI4, ST36, and left unilateral auriculotherapy: Thalamus point or Subcortex (MA-AT2), Lung (MA-IC1), ear Shen Men (MA-TF 1), Stomach (MA-IC 6), Face (LO-5). No adverse events were reported. After five sessions the patient had been symptoms-free for the last two weeks, without need of pharmacological treatment and reaching a VAS score of 0 . Hence he has been discharged (Figure 1A). Quality of life evaluation with the SF-12 questionnaire showed a slight improvement of the physical score (from 49.16 to 53.11), while the mental score has changed from 33.76 to 29.61 . Results were consistent at ten weeks follow up.

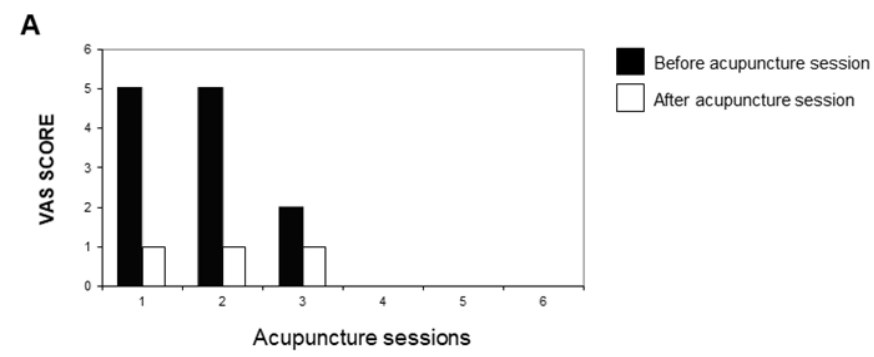

Figure IA The graph represents the VAS score before and after every acupuncture session over six weeks of treatment.

One year follow-up was carried out. The patient showed the same symptoms but with a low intensity (VAS score 3); applying the same pattern of acupuncture points, previously described, once a week for a month, the symptoms completely regressed (Figure 1B). Quality of life evaluation with the SF-12 questionnaire showed again a slight improvement in the physical score (from 28,44 to 42,55 ), and in the mental score (from 24,90 to 34,89 ).

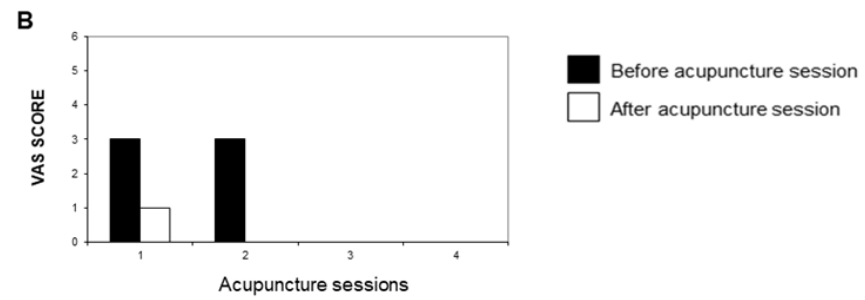

Figure IB One year follow-up. The graph represents the VAS score before and after every acupuncture session over four weeks of treatment.

\section{Discussion}

Facial pain remains a diagnostic and therapeutic challenge for both clinicians and patients. Patients suffering from facial pain generally undergo multiple consultations with different specialists. Trigeminal neuralgia is the most common cranial neuralgia and its treatment initially consists of carbamazepine or other antiepileptic drugs. ${ }^{12}$ However, many patients experience significant side-effects. Surgical micro vascular decompression and gamma knife radiation have been shown to treat "classic" cases with the best long term remission. Less invasive techniques include balloon dilatation and glycerol lysis of the Gasser's ganglion. ${ }^{13,14}$ The facial anatomical abnormalities characterizing PS along with the surgeries which the patient underwent, may have caused damage, entrapment or suffering of the 5th cranial nerve. Indeed, the symptoms and their distribution were consistent with a neuropathic involvement of the second trigeminal branch, even if no RMI confirmation had been taken previously. As the first line treatments have not proven helpful, the patient had been referred to our UIM. Recent literature shows that traditional acupuncture whether combined with auriculotherapy yields a substantial improvement in TN pain with negligible side-effects..$^{15,16}$ Accordingly, the present case describes the complete recovery from facial pain and associated symptoms, obtained with both types of acupuncture.

In agreement with the general consensus ${ }^{17}$ chosen acupoints were local points, in proximity to the affected branch of the trigeminal nerve, combined with distal points according to the affected channel. The local points used are known to exert an effect by expelling the wind and clearing the heat of the head, regarding the eyes, opening the nasal passages, reducing swellings and alleviate pain. Yintang has been used both to eliminate the wind, to alleviates pain and for his specific action on the Shen and its harmonising effect. The distal points LI4, and ST36, are thought to circulate Qi, activate blood, and disperse blood stasis. Auriculotherapy has also been used, focusing on reducing the physical emotional components of pain sensation (Shenmen, Thalamus); needling the auricular reflex point of the Face and Stomach were meant to alleviate pain in the corresponding area (face) and the structures found along the Stomach meridian (face), whereas the Lung point specifically relieves trigeminal neuralgia and dispel invasive wind.

Loss of left nasal foreign body sensation and pain relief was achieved immediately during the first session of acupuncture, as the VAS score decreased considerably, while complete recovery was obtained after the third session and sustained up to ten weeks follow up. The slight variation observed in both physical and mental component of the SF-12, ${ }^{18}$ might represent an unexpected or contradictory result. Though, an overall clinical assessment should be considered: the facial and body abnormalities of the Pfeiffer syndrome have a severe impact on the psychological life of a young man that 
could include feelings of isolation, depression, inadequacy, anger, which can hardly be modified by improving one symptom. Selfordered suspension of pharmacological treatment and the tendency of the patient to postpone the medical follow up were taken as an indicator of the general physical improvement. A follow-up after one year showed a relapse of symptoms at a much lower intensity; indeed, a shorter cycle of acupuncture was needed for remission. Even if the patient's perception of his physical and mental status were poorer than a year before, four acupuncture sessions were enough to ameliorate both components and to treat the pain.

\section{Conclusion}

Therefore, we can conclude that acupuncture is an effective treatment in relieving $\mathrm{TN}$ pain even in patients with congenital anatomical disharmonies, leading to reduction or withdrawal of previous analgesic medication. This improvement can be sustained up to a year and can be eventually restored with a second boost of acupuncture.

\section{Acknowledgments}

None.

\section{Conflicts of interest}

Author declares that there are no conflicts of interest.

\section{Funding}

None.

\section{References}

1. van Hecke O, Austin SK, Khan RA, et al. Neuropathic pain in the general population: a systematic review of epidemiological studies. Pain. 2014;155(4):654-662

2. Merskey H, Bogduk N. Classification of chronic pain. Descriptions of chronic pain syndromes and definitions of pain terms. Seattle, WA: IASP Press; 1994. p. 59-71

3. Brisman R. Typical versus atypical trigeminal neuralgia and other factors that may affect results of neurosurgical treatment. World Neurosurg. 2013;79(5-6):649-650.

4. Tölle T, Dukes E, Sadosky A. Patient Burden of Trigeminal Neuralgia: Results from a Cross-Sectional Survey of Health State Impairment and Treatment Patterns in Six European Countries. Pain Pract. 2006;6(30:153-160
5. Padfield D. The Patient's Journey Through Trigeminal Neuralgia. Pain Clin Updates. 2014;22(1):1-8.

6. Cohen MM. Pfeiffer syndrome update, clinical subtypes, and guidelines for differential diagnosis. Am J Med Genet. 1993;45(3):300-307.

7. Fearon JA, Rhodes J. Pfeiffer syndrome: a treatment evaluation. Plast Reconstr Surg. 2009;123(5):1560-1569.

8. Hockstein NG, McDonald-McGinn D, Zackai E, et al. Tracheal anomalies in Pfeiffer syndrome. Arch Otolaryngol Head Neck Surg. 2004;130(11):1298-1302.

9. Zhang ZJ, Wang XM, McAlonan GM. Neural Acupuncture Unit: A New Concept for Interpreting Effects and Mechanisms of Acupuncture. Evid Based Complement Alternat Med. 2012;23.

10. Irnich D, Winklmeier S, Beyer A, et al. Electric stimulation acupuncture in peripheral neuropathic pain syndromes. Clinical pilot study on analgesic effectiveness. Schmerz. 2002;16(2):114-120.

11. Ahn CB, Lee SJ, Lee JC, et al. A clinical pilot study comparing traditional acupuncture to combined acupuncture for treating headache, trigeminal neuralgia and retro-auricular pain in facial palsy. $J$ Acupunct Meridian Stud. 2011;4(1):29-43.

12. Di Stefano G, Truini A. Pharmacological treatment of trigeminal neuralgia. Expert Rev Neurother. 2017;17(10):1003-1011.

13. Chavin JM. Cranial neuralgias and headaches associated with cranial vascular disorders. Otolaryngol Clin North Am. 2003;36(60:1079-1093.

14. Cruccu G. Trigeminal neuralgia. Continuum (Minneap Minn). 2017;23:396-320.

15. He L, Zhang XM. Clinical effect of sphenopalatine ganglion needling in treating primary trigeminal neuralgia of Liver-Yang upsurge syndrome type. Chin J Integr Med. 2012;18(30:214-218.

16. Zhu J, Arsovska B, Kozovska K, et al. Two Case Reports: Acupuncture Treatment for Trigeminal Neuralgia. American Journal of Health Research. 2017;5(3):54-56.

17. Kwokming C. Neuroanatomical basis of acupuncture treatment for some common illnesses. Acupunct Med. 2009;27(4):61-64.

18. Gandek B, Ware JE, Aaronson NK, et al. Cross-validation of item selection and scoring for the SF-12 Health Survey in nine countries: Results from the IQOLA Project. International Quality of Life Assessment. J Clin Epidemiol. 1998;51(11):1171-7118. 